\title{
UJI KESTABILAN ASIMTOTIK SISTEM LINIER POSITIF MENGGUNAKAN KRITERIA LYAPUNOV
}

\author{
ELFID SYUKRIATI EMRI, ZULAKMAL \\ Jurusan Matematika, \\ Fakultas Matematika dan Ilmu Pengetahuan Alam, Universitas Andalas, \\ Kampus UNAND Limau Manis Padang, Indonesia. \\ email : elfidsyukriatiemri@gmail.com
}

\begin{abstract}
Suatu sistem linier dikatakan positif jika untuk setiap keadaan awal nonnegatif, maka trajektori dari sistem linier tersebut adalah non-negatif dengan berlalunya waktu. Suatu sistem linier (tanpa batas kepositifan) dikatakan stabil asimtotik jika bagian rill dari semua nilai eigennya adalah negatif. Pada tulisan ini dikaji kriteria uji ketsabilan asimtotik sistem linier postif menggunakan kriteria Lyapunov.
\end{abstract}

Kata Kunci: Sistem linier positif, kestabilan asimtotik, kriteria Lyapunov

\section{Pendahuluan}

Diberikan suatu sistem linier sebagai berikut :

$$
\dot{\mathbf{x}}=A \mathbf{x}(t), \mathbf{x}(0)=\mathbf{x}_{0}
$$

dimana $A \in \mathbb{R}^{n \times n}, \mathbf{x} \in \mathbb{R}^{n}$ dan $t \in \mathbb{R}_{+}$. Sistem (1.1) dikatakan positif jika untuk setiap keadaan awal $\mathbf{x}_{0} \in \overline{\mathbb{R}}_{+}^{n}$ dan $t \geq 0$ maka solusi dari (1.1) adalah non-negatif, yaitu $\mathbf{x}(t) \in \overline{\mathbb{R}}_{+}^{n}$. Kajian dari sistem linier dimulai dengan memperkenalkan terminologi titik tetap. Suatu titik $\mathbf{x}^{*}$ dikatakan titik tetap dari sistem (1.1) jika $A \mathbf{x}^{*}=\mathbf{0}$. Dalam makalah ini dikaji masalah kestabilan asimtotik dari sistem linier dengan asumsi bahwa sistem (1.1) adalah positif dengan menggunakan kriteria kestabilan Lyapunov.

\section{Pembahasan}

Suatu matriks $A \in \mathbb{R}^{n \times n}$ dikatakan simetris jika $A^{T}=A$. Suatu matriks simetris $A \in \mathbb{R}^{n \times n}$ dikatakan definit positif, dinotasikan dengan $A>0$, jika $\mathbf{x}^{T} A \mathbf{x}>0$ untuk setiap vektor $\mathbf{x} \neq 0$.

Teorema berikut diperlukan untuk membuktikan hasil utama.

Teorema 2.1. [1] Matriks simetris A adalah definit positif jika dan hanya jika semua nilai eigen $A$ adalah positif.

Matriks $A=\left[a_{i j}\right] \in \mathbb{R}^{n \times n}$ dikatakan matriks Metzler jika entri-entri selain entri diagonal utamanya adalah non-negatif, yaitu $a_{i j} \geq 0$ untuk $i \neq j, i, j=1,2, \cdots, n$ [5]. Matriks $A=\left[a_{i j}\right] \in \mathbb{R}^{n \times n}$ dikatakan matriks Hurwitz jika setiap bagian real dari nilai eigennya adalah negatif [2]. 
Teorema 2.2. [3] Sistem (1.1) adalah positif jika dan hanya jika matriks $A \in \mathcal{M}_{n}$.

Teorema 2.3. [6] Syarat perlu dan cukup untuk suatu titik tetap sistem (1.1) menjadi stabil asimtotik yaitu untuk setiap bagian real nilai eigen dari A bernilai negatif (Hurwitz).

Misalkan $V: \mathbb{R}^{n} \rightarrow \mathbb{R}^{n}$ dan $\mathbf{x}^{*}$ adalah titik tetap dari sistem (1.1). Fungsi $V$ dikatakan fungsi Lyapunov jika $V\left(\mathbf{x}^{*}\right)=0$ dan $V(\mathbf{x})>0$ untuk $\mathbf{x} \neq \mathbf{x}^{*}$ [2]. Teorema Lyapunov dalam [4] menyatakan bahwa sistem (1.1) adalah stabil asimtotik jika $\dot{V}(\mathbf{x})=V^{\prime}(\mathbf{x}) A \mathbf{x} \in \mathbb{R}_{-}$.

Teorema 2.4. [4] Perhatikan sistem (1.1) dengan $A \in \mathcal{M}_{n}$. Jika terdapat vektorvektor $\mathbf{p}, \mathbf{r} \in \mathbb{R}_{+}^{n}$ sedemikian sehingga

$$
\mathbf{0}=A^{T} \mathbf{p}+\mathbf{r},
$$

maka $A$ adalah stabil asimtotik.

Bukti. Misalkan terdapat vektor-vektor $\mathbf{p}, \mathbf{r} \in \mathbb{R}_{+}^{n}$ sedemikian sehingga

$$
\mathbf{0}=A^{T} \mathbf{p}+\mathbf{r},
$$

dan perhatikan bahwa fungsi Lyapunov

$$
V(\mathbf{x})=\mathbf{p}^{T} \mathbf{x}, \mathbf{x} \in \mathbb{R}_{+}^{n},
$$

maka

$$
\dot{V}(\mathbf{x})=\mathbf{p}^{T} \dot{\mathbf{x}}=\mathbf{p}^{T} A \mathbf{x}=-\mathbf{r}^{T} \mathbf{x}<0 .
$$

Sehingga berdasarkan Teorema Lyapunov, $A$ adalah stabil asimtotik.

Akibat 2.5. [4] Misalkan $A \in \mathcal{M}_{n}$, maka $A$ adalah Hurwitz jika dan hanya jika terdapat suatu vektor $\mathbf{p} \in \mathbb{R}_{+}^{n}$ sedemikian sehingga $A^{T} \mathbf{p} \in \mathbb{R}_{-}^{n}$.

Bukti. $(\Rightarrow)$ Misal $A$ adalah Hurwitz. Berdasarkan Teorema 2.4 terdapat vektorvektor $\mathbf{p}, \mathbf{r} \in \mathbb{R}_{+}^{n}$ sedemikian sehingga

$$
\mathbf{0}=A^{T} \mathbf{p}+\mathbf{r} .
$$

Karena $\mathbf{r} \in \mathbb{R}_{+}^{n}$, maka mestilah $A^{T} \mathbf{p} \in \mathbb{R}_{-}^{n}$.

$(\Leftarrow)$ Misalkan terdapat $\mathbf{p} \in \mathbb{R}_{+}^{n}$ sedemikian sehingga $A^{T} \mathbf{p} \in \mathbb{R}_{-}^{n}$. Karena $A^{T} \mathbf{p} \in \mathbb{R}_{-}^{n}$, maka terdapat vektor $\mathbf{r} \in \mathbb{R}_{+}^{n}$ sedemikian sehingga

$$
\mathbf{0}=A^{T} \mathbf{p}+\mathbf{r} .
$$

Berdasarkan Teorema 2.4 maka mestilah $A$ stabil asimtotik dan berdasarkan Teorema 2.3 A adalah Hurwitz.

Lema 2.6. [4] Misal $\hat{A} \in \mathcal{M}_{n}$ sedemikian sehingga $\hat{A} \boldsymbol{e} \in \mathbb{R}_{-}^{n}$ dan $\hat{A}^{T} \boldsymbol{e} \in \mathbb{R}_{-}^{n}$ maka $\hat{A}+\hat{A}^{T} \in \mathbb{R}_{-}^{n \times n}$ 
Bukti. Misalkan

$$
\hat{A}=\left[\begin{array}{cccc}
a_{11} & a_{12} & \cdots & a_{1 n} \\
a_{21} & a_{22} & \cdots & a_{2 n} \\
\vdots & \vdots & \ddots & \vdots \\
a_{n 1} & a_{n 2} & \cdots & a_{n n}
\end{array}\right] \in \mathcal{M}_{n}
$$

maka $\hat{A} \mathbf{e} \in \mathbb{R}_{-}^{n}$ dan $\hat{A}^{T} \mathbf{e} \in \mathbb{R}_{-}^{n}$.

Akibatnya

$$
\begin{aligned}
\hat{A}+\hat{A}^{T}= & {\left[\begin{array}{cccc}
a_{11} & a_{12} & \cdots & a_{1 n} \\
a_{21} & a_{22} & \cdots & a_{2 n} \\
\vdots & \vdots & \ddots & \vdots \\
a_{n 1} & a_{n 2} & \cdots & a_{n n}
\end{array}\right]+\left[\begin{array}{cccc}
a_{11} & a_{21} & \cdots & a_{n 1} \\
a_{12} & a_{22} & \cdots & a_{n 2} \\
\vdots & \vdots & \ddots & \vdots \\
a_{1 n} & a_{2 n} & \cdots & a_{n n}
\end{array}\right] } \\
= & {\left[\begin{array}{cccc}
a_{11}+a_{11} & a_{12}+a_{21} & \cdots & a_{1 n}+a_{n 1} \\
a_{21}+a_{12} & a_{22}+a_{22} & \cdots & a_{2 n}+a_{n 2} \\
\vdots & \vdots & \ddots & \vdots \\
a_{n 1}+a_{1 n} & a_{n 2}+a_{2 n} & \cdots & a_{n n}+a_{n n}
\end{array}\right] \in \mathcal{M}_{n}, }
\end{aligned}
$$

dan

$$
\begin{aligned}
\left(\hat{A}+\hat{A}^{T}\right) \mathbf{e}= & {\left[\begin{array}{cccc}
a_{11}+a_{11} & a_{12}+a_{21} & \cdots & a_{1 n}+a_{n 1} \\
a_{21}+a_{12} & a_{22}+a_{22} & \cdots & a_{2 n}+a_{n 2} \\
\vdots & \vdots & \ddots & \vdots \\
a_{n 1}+a_{1 n} & a_{n 2}+a_{2 n} & \cdots & a_{n n}+a_{n n}
\end{array}\right]\left[\begin{array}{c}
1 \\
1 \\
\vdots \\
1
\end{array}\right] } \\
= & {\left[\begin{array}{c}
\left(a_{11}+a_{11}\right)+\left(a_{12}+a_{21}\right)+\cdots+\left(a_{1 n}+a_{n 1}\right) \\
\left(a_{21}+a_{12}\right)+\left(a_{22}+a_{22}\right)+\cdots+\left(a_{2 n}+a_{n 2}\right) \\
\vdots \\
\left(a_{n 1}+a_{1 n}\right)+\left(a_{n 2}+a_{2 n}\right)+\cdots+\left(a_{n n}+a_{n n}\right)
\end{array}\right] \in \mathbb{R}_{-}^{n} . }
\end{aligned}
$$

Berdasarkan Akibat $2.5 \hat{A}+\hat{A}^{T}$ adalah Hurwitz. Karena $\hat{A}+\hat{A}^{T}$ adalah simetris, mengakibatkan $\hat{A}+\hat{A}^{T} \in \mathbb{R}_{-}^{n \times n}$.

Teorema 2.7. [4] Perhatikan sistem yang diberikan oleh (1.1) dimana $A \in \mathcal{M}_{n}$, maka sistem (1.1) adalah stabil asimtotik jika dan hanya jika terdapat suatu matriks diagonal positif $P \in \mathbb{R}^{n \times n}$ dan suatu matriks definit positif $R \in \mathbb{R}^{n \times n}$ sedemikian sehingga

$$
0=A^{T} P+P A+R
$$

Bukti. $(\Rightarrow)$ Misalkan sistem (1.1) stabil asimtotik dan $A \in \mathcal{M}_{n}$. Akan dibuktikan terdapat suatu matriks diagonal positif $P \in \mathbb{R}^{n \times n}$ dan matriks definit positif $R$ $\in \mathbb{R}^{n \times n}$ sedemikian sehingga (2.2) terpenuhi.

Karena sistem (1.1) adalah stabil asimtotik dan $A \in \mathcal{M}_{n}$, maka terdapat vektorvektor $\mathbf{p}, \mathbf{r} \in \mathbb{R}_{+}^{n}$ sedemikian sehingga (2.1) berlaku. Berdasarkan Akibat 2.5 ada suatu vektor $\mathbf{q} \in \mathbb{R}_{+}^{n}$ sedemikian sehingga $A^{T} \mathbf{q} \in \mathbb{R}_{-}^{n}$. 
28 Elfid Syukriati Emri, Zulakmal

Misalkan

$$
\mathbf{q}=\left[\begin{array}{c}
q_{1} \\
q_{2} \\
\vdots \\
q_{n}
\end{array}\right]
$$

$Q=\operatorname{diag}\left[q_{1}, q_{2}, \cdots, q_{n}\right], q_{i}$ adalah komponen ke- $i$ dari $\mathbf{q}$ dan $\mathbf{e}=\left[\begin{array}{llll}1 & 1 & \cdots & 1\end{array}\right]^{T}$ maka

$$
\begin{aligned}
A^{T} Q \mathbf{e}= & {\left[\begin{array}{cccc}
a_{11} & a_{21} & \cdots & a_{n 1} \\
a_{12} & a_{22} & \cdots & a_{n 2} \\
\vdots & \vdots & \ddots & \vdots \\
a_{1 n} & a_{2 n} & \cdots & a_{n n}
\end{array}\right]\left[\begin{array}{cccc}
q_{1} & 0 & \cdots & 0 \\
0 & q_{2} & \cdots & 0 \\
\vdots & \vdots & \ddots & \vdots \\
0 & 0 & \cdots & q_{n}
\end{array}\right]\left[\begin{array}{c}
1 \\
1 \\
\vdots \\
1
\end{array}\right] } \\
= & {\left[\begin{array}{c}
a_{11} q_{1}+a_{21} q_{2}+\cdots+a_{n 1} q_{n} \\
a_{12} q_{1}+a_{22} q_{2}+\cdots+a_{n 2} q_{n} \\
\vdots \\
a_{1 n} q_{1}+a_{2 n} q_{2}+\cdots+a_{n n} q_{n}
\end{array}\right] } \\
= & A^{T} \mathbf{q} .
\end{aligned}
$$

Karena $A^{T} Q e=A^{T} \mathbf{q}$ maka $A^{T} Q e \in \mathbb{R}_{-}^{n}$. Hal ini juga mengakibatkan $Q A \in \mathcal{M}_{n}$ dan Hurwitz. Misal $A^{T} Q e \in \mathbb{R}_{-}^{n}$, perhatikan bahwa

$$
\begin{aligned}
\left(A^{T} Q\right)^{T}= & Q A \\
= & {\left[\begin{array}{cccc}
q_{1} & 0 & \cdots & 0 \\
0 & q_{2} & \cdots & 0 \\
\vdots & \vdots & \ddots & \vdots \\
0 & 0 & \cdots & q_{n}
\end{array}\right]\left[\begin{array}{cccc}
a_{11} & a_{12} & \cdots & a_{1 n} \\
a_{21} & a_{22} & \cdots & a_{2 n} \\
\vdots & \vdots & \ddots & \vdots \\
a_{n 1} & a_{n 2} & \cdots & a_{n n}
\end{array}\right] } \\
= & {\left[\begin{array}{cccc}
q_{1} a_{11} & q_{1} a_{12} & \cdots & q_{1} a_{1 n} \\
q_{2} a_{21} & q_{2} a_{22} & \cdots & q_{2} a_{2 n} \\
\vdots & \vdots & \ddots & \vdots \\
q_{n} a_{n 1} & q_{n} a_{n 2} & \cdots & q_{n} a_{n n}
\end{array}\right] . }
\end{aligned}
$$

Karena $q_{i} \in \mathbb{R}_{+}$dan $A \in \mathcal{M}_{n}$, maka $Q A$ juga $\in \mathcal{M}_{n}$. Akan ditunjukkan $Q A$ adalah Hurwitz, berdasarkan Akibat 2.5 akan ditunjukkan $A^{T} Q \mathbf{p} \in \mathbb{R}_{-}^{n}$. Misalkan

$$
\mathbf{p}=\mathbf{e}
$$


maka

$$
\begin{aligned}
A^{T} Q \mathbf{p}= & {\left[\begin{array}{cccc}
a_{11} & a_{21} & \cdots & a_{n 1} \\
a_{12} & a_{22} & \cdots & a_{n 2} \\
\vdots & \vdots & \ddots & \vdots \\
a_{1 n} & a_{2 n} & \cdots & a_{n n}
\end{array}\right]\left[\begin{array}{cccc}
q_{1} & 0 & \cdots & 0 \\
0 & q_{2} & \cdots & 0 \\
\vdots & \vdots & \ddots & \vdots \\
0 & 0 & \cdots & q_{n}
\end{array}\right]\left[\begin{array}{c}
1 \\
1 \\
\vdots \\
1
\end{array}\right] } \\
= & {\left[\begin{array}{c}
a_{11} q_{1}+a_{21} q_{2}+\cdots+a_{n 1} q_{n} \\
a_{12} q_{1}+a_{22} q_{2}+\cdots+a_{n 2} q_{n} \\
\vdots \\
a_{1 n} q_{1}+a_{2 n} q_{2}+\cdots+a_{n n} q_{n}
\end{array}\right] \in \mathbb{R}_{-}^{n} . }
\end{aligned}
$$

Pilih

$$
\mathbf{r}=-A^{T} Q \mathbf{p} \in \mathbb{R}_{+}^{n}
$$

maka berlaku persamaan (2.1). Hal ini juga mengakibatkan $A^{T} Q \in \mathcal{M}_{n}$ dan Hurwitz. Berdasarkan Akibat 2.5, misal ada suatu vektor $\mathbf{m} \in \mathbb{R}_{+}^{n}$ dengan $M=\operatorname{diag}\left[m_{1}, \cdots, m_{n}\right], m_{i}$ adalah komponen ke- $i$ dari $\mathbf{m}$ sedemikian sehingga $Q A \mathbf{m} \in \mathbb{R}_{-}^{n}$.

Akibatnya

$$
\begin{aligned}
Q A M \mathbf{e}= & {\left[\begin{array}{cccc}
q_{1} & 0 & \cdots & 0 \\
0 & q_{2} & \cdots & 0 \\
\vdots & \vdots & \ddots & \vdots \\
0 & 0 & \cdots & q_{n}
\end{array}\right]\left[\begin{array}{cccc}
a_{11} & a_{12} & \cdots & a_{1 n} \\
a_{21} & a_{22} & \cdots & a_{2 n} \\
\vdots & \vdots & \ddots & \vdots \\
a_{n 1} & a_{n 2} & \cdots & a_{n n}
\end{array}\right]\left[\begin{array}{cccc}
m_{1} & 0 & \cdots & 0 \\
0 & m_{2} & \cdots & 0 \\
\vdots & \vdots & \ddots & \vdots \\
0 & 0 & \cdots & m_{n}
\end{array}\right]\left[\begin{array}{c}
1 \\
1 \\
\vdots \\
1
\end{array}\right] } \\
= & {\left[\begin{array}{c}
q_{1} a_{11} m_{1}+q_{1} a_{12} m_{2}+\cdots+q_{1} a_{1 n} m_{n} \\
q_{2} a_{21} m_{1}+q_{2} a_{22} m_{2}+\cdots+q_{2} a_{2 n} m_{n} \\
\vdots \\
q_{n} a_{n 1} m_{1}+q_{n} a_{n 2} m_{2}+\cdots+q_{n} a_{n n} m_{n}
\end{array}\right] \in \mathbb{R}_{-}^{n} . }
\end{aligned}
$$

Karena $A^{T} Q e \in \mathbb{R}_{-}^{n}$, hal ini mengakibatkan $M A^{T} Q e \in \mathbb{R}_{-}^{n}$. Misal $\hat{A}=Q A M$, berdasarkan Lemma 2.6 maka

$$
M A^{T} Q+Q A M \in \mathbb{R}_{-}^{n \times n}
$$

ini ekuivalen dengan

$$
A^{T} Q M^{-1}+M^{-1} Q A \in \mathbb{R}_{-}^{n \times n}
$$

Misal $P=Q M^{-1}$ dan $R=-\left(A^{T} P+P A\right)$, maka (2.2) terpenuhi.

$(\Leftarrow)$ Misal ada suatu matriks diagonal positif $P \in \mathbb{R}^{n \times n}$ dan matriks definit positif $R \in \mathbb{R}^{n \times n}$ sedemikian sehingga (2.2) terpenuhi. Akan dibuktikan sistem (1.1) stabil asimtotik yaitu dengan menunjukkan $\dot{V}(\mathbf{x})<0$. Misalkan $V(\mathbf{x})=\mathbf{x}^{T} P \mathbf{x}$ adalah 
suatu fungsi Lyapunov, maka

$$
\begin{aligned}
\dot{V}(\mathbf{x}) & =\dot{\mathbf{x}}^{T} P \mathbf{x}+\mathbf{x}^{T} P \dot{\mathbf{x}} \\
& =(A \mathbf{x})^{T} P \mathbf{x}+\mathbf{x}^{T} P(A \mathbf{x}) \\
& =\mathbf{x}^{T} A^{T} P \mathbf{x}+\mathbf{x}^{T} P A \mathbf{x} \\
& =\mathbf{x}^{T}\left(A^{T} P+P A\right) \mathbf{x} \\
& =\mathbf{x}^{T}(-R) \mathbf{x} \\
& =-\mathbf{x}^{T} R \mathbf{x}<0 .
\end{aligned}
$$

Karena $R$ adalah definit positif, maka $\dot{V}(\mathbf{x})=-\mathbf{x}^{T} R \mathbf{x} \in \mathbb{R}_{-}$. Akibatnya sistem (1.1) adalah stabil asimtotik.

Sebagai suatu ilustrasi, perhatikan contoh berikut.

Contoh 2.8. Diberikan sistem (1.1) dengan

$$
\left[\begin{array}{l}
\dot{x}_{1}(t) \\
\dot{x}_{2}(t)
\end{array}\right]=\left[\begin{array}{cc}
-2 & 0 \\
0 & -1
\end{array}\right]\left[\begin{array}{l}
x_{1} \\
x_{2}
\end{array}\right] .
$$

Pilih suatu matriks $\mathrm{R}$ definit positif, yaitu

$$
R=\left[\begin{array}{cc}
2 & -1 \\
-1 & 2
\end{array}\right]
$$

Akan dicari suatu matriks $\mathrm{P}$ diagonal positif, sehingga $A^{T} P+P A=-R$ maka,

$$
\begin{aligned}
{\left[\begin{array}{cc}
-2 & 0 \\
0 & -1
\end{array}\right]\left[\begin{array}{cc}
p_{1} & 0 \\
0 & p_{2}
\end{array}\right]+\left[\begin{array}{cc}
p_{1} & 0 \\
0 & p_{2}
\end{array}\right]\left[\begin{array}{cc}
-2 & 0 \\
0 & -1
\end{array}\right] } & =-\left[\begin{array}{cc}
2 & -1 \\
-1 & 2
\end{array}\right] \\
{\left[\begin{array}{cc}
-2 p_{1} & 0 \\
0 & -p_{2}
\end{array}\right]+\left[\begin{array}{cc}
-2 p_{1} & 0 \\
0 & -p_{2}
\end{array}\right] } & =-\left[\begin{array}{cc}
2 & -1 \\
-1 & 2
\end{array}\right] \\
{\left[\begin{array}{cc}
-4 p_{1} & 0 \\
0 & -2 p_{2}
\end{array}\right] } & =-\left[\begin{array}{cc}
2 & -1 \\
-1 & 2
\end{array}\right]
\end{aligned}
$$

diperoleh

$$
\begin{aligned}
& -4 p_{1}=-2 \Rightarrow p_{1}=\frac{1}{2} \\
& -2 p_{2}=-2 \Rightarrow p_{2}=1
\end{aligned}
$$

sehingga diperoleh matriks

$$
P=\left[\begin{array}{ll}
\frac{1}{2} & 0 \\
0 & 1
\end{array}\right]
$$

dimana matriks $P$ merupakan matriks diagonal positif, sehingga sistem (1.1) merupakan sistem yang stabil asimtotik.

Contoh 2.9. Diberikan sistem (1.1) dengan

$$
\left[\begin{array}{l}
\dot{x}_{1}(t) \\
\dot{x}_{2}(t) \\
\dot{x}_{3}(t) \\
\dot{x}_{4}(t)
\end{array}\right]=\left[\begin{array}{cccc}
-2 & 0 & 0 & 0 \\
0 & -2 & 1 & 0 \\
0 & 0 & -2 & 1 \\
0 & 2 & 0 & -3
\end{array}\right]\left[\begin{array}{l}
x_{1} \\
x_{2} \\
x_{3} \\
x_{4}
\end{array}\right] .
$$


Pilih suatu matriks R definit positif, yaitu

$$
R=\left[\begin{array}{cccc}
4 & 0 & 0 & 0 \\
0 & 4 & -1 & -2 \\
0 & -1 & 4 & -1 \\
0 & -2 & -1 & 6
\end{array}\right]
$$

Akan dicari suatu matriks $\mathrm{P}$ diagonal positif, sehingga $A^{T} P+P A=-R$ maka,

$$
\begin{aligned}
& {\left[\begin{array}{cccc}
-2 & 0 & 0 & 0 \\
0 & -2 & 0 & 2 \\
0 & 1 & -2 & 0 \\
0 & 0 & 1 & -3
\end{array}\right]\left[\begin{array}{cccc}
p_{1} & 0 & 0 & 0 \\
0 & p_{2} & 0 & 0 \\
0 & 0 & p_{3} & 0 \\
0 & 0 & 0 & p_{4}
\end{array}\right]+\left[\begin{array}{cccc}
p_{1} & 0 & 0 & 0 \\
0 & p_{2} & 0 & 0 \\
0 & 0 & p_{3} & 0 \\
0 & 0 & 0 & p_{4}
\end{array}\right]\left[\begin{array}{cccc}
-2 & 0 & 0 & 0 \\
0 & -2 & 1 & 0 \\
0 & 0 & -2 & 1 \\
0 & 2 & 0 & -3
\end{array}\right]} \\
& =-\left[\begin{array}{cccc}
4 & 0 & 0 & 0 \\
0 & 4 & -1 & -2 \\
0 & -1 & 4 & -1 \\
0 & -2 & -1 & 6
\end{array}\right] \\
& {\left[\begin{array}{cccc}
-2 p_{1} & 0 & 0 & 0 \\
0 & -2 p_{2} & 0 & 2 p_{4} \\
0 & p_{2} & -2 p_{3} & 0 \\
0 & 0 & p_{3} & -3 p_{4}
\end{array}\right]+\left[\begin{array}{cccc}
-2 p_{1} & 0 & 0 & 0 \\
0 & -2 p_{2} & p_{2} & 0 \\
0 & 0 & -2 p_{3} & p_{3} \\
0 & 2 p_{4} & 0 & -3 p_{4}
\end{array}\right]=-\left[\begin{array}{cccc}
4 & 0 & 0 & 0 \\
0 & 4 & -1 & -2 \\
0 & -1 & 4 & -1 \\
0 & -2 & -1 & 6
\end{array}\right]} \\
& {\left[\begin{array}{cccc}
-4 p_{1} & 0 & 0 & 0 \\
0 & -4 p_{2} & p_{2} & 2 p_{4} \\
0 & p_{2} & -4 p_{3} & p_{3} \\
0 & 2 p_{4} & p_{3} & -6 p_{4}
\end{array}\right]=-\left[\begin{array}{cccc}
4 & 0 & 0 & 0 \\
0 & 4 & -1 & -2 \\
0 & -1 & 4 & -1 \\
0 & -2 & -1 & 6
\end{array}\right]}
\end{aligned}
$$

diperoleh

$$
\begin{aligned}
& -4 p_{1}=-4 \Rightarrow p_{1}=1 \\
& -4 p_{2}=-4 \Rightarrow p_{2}=1 \\
& -4 p_{3}=-4 \Rightarrow p_{3}=1 \\
& -6 p_{4}=-6 \Rightarrow p_{4}=1
\end{aligned}
$$

sehingga diperoleh matriks

$$
P=\left[\begin{array}{llll}
1 & 0 & 0 & 0 \\
0 & 1 & 0 & 0 \\
0 & 0 & 1 & 0 \\
0 & 0 & 0 & 1
\end{array}\right]
$$

dimana matriks $P$ merupakan matriks diagonal positif sehingga sistem (1.1) merupakan sistem yang stabil asimtotik. 


\section{Kesimpulan}

Dari uraian pada pembahasan dapat dilihat bahwa kestabilan dari sistem (1.1) tergantung pada solusi dari persamaan (2.2)

$$
0=A^{T} P+P A+R
$$

yaitu jika terdapat suatu matriks diagonal positif $P \in \mathbb{R}^{n \times n}$ sedemikian sehingga $R \in \mathbb{R}^{n \times n}$ adalah suatu matriks definit positif maka sistem (1.1) adalah stabil asimtotik.

\section{Ucapan Terima kasih}

Penulis mengucapkan terima kasih kepada bapak Prof. Dr. Muhafzan selaku dosen pembimbing dan bapak Dr. Mahdhivan Syafwan, ibu Riri Lestari, M.Si, bapak Dr. Effendi selaku dosen penguji yang telah memberikan masukan dan saran sehingga makalah ini dapat diselesaikan dengan baik.

\section{Daftar Pustaka}

[1] Anton, H. 2005. Elementary Linear Algebra. Ninth Edition. Wiley.

[2] Antsaklis, P. J and Michel, A. N. 2007. A Linier Systems Primer. Birkhauser. Boston.

[3] Farina, L. and Rinaldi, S. 2000. Positive Linear Systems : Theory and Aplications. Wiley. New York.

[4] Haddad, W. M and Chellaboina, V and Hui, Qing 2010. Nonnegative and Compartmental Dynamical Systems. Princeton University Press. Princeton and Oxford.

[5] Kaczorek, T. 2001. Positive 1D and 2D Systems Metzler Matrices. SpringerVerlag Berlin Heidelberg.

[6] Luenburger, David G. 1979. Introduction to Dynamic System: Theory, Models and Aplication. Wiley. New York. 